\title{
Improved Visibility of Road Scene Images under Heterogeneous Fog
}

\author{
Jean-Philippe Tarel Nicolas Hautière \\ Université Paris-Est, LEPSIS, INRETS-LCPC \\ 58 Boulevard Lefèbvre, F-75015 Paris, \\ France \\ tarelelcpc.fr hautiereelcpc.fr
}

\author{
Aurélien Cord Dominique Gruyer \\ Houssam Halmaoui \\ UniverSud, LIVIC, INRETS-LCPC \\ 14 route de la Minière, F-78000 Versailles, \\ France \\ cordelcpc.fr gruyereinrets.fr \\ halmaouidlcpc.fr
}

\begin{abstract}
One source of accidents when driving a vehicle is the presence of homogeneous and heterogeneous fog. Fog fades the colors and reduces the contrast of the observed objects with respect to their distances. Various camera-based Advanced Driver Assistance Systems (ADAS) can be improved if efficient algorithms are designed for visibility enhancement of road images. The visibility enhancement algorithm proposed in [1] is not dedicated to road images and thus it leads to limited quality results on images of this kind. In this paper, we interpret the algorithm in [1] as the inference of the local atmospheric veil subject to two constraints. From this interpretation, we propose an extended algorithm which better handles road images by taking into account that a large part of the image can be assumed to be a planar road. The advantages of the proposed local algorithm are its speed, the possibility to handle both color images or gray-level images, and its small number of parameters. A comparative study and quantitative evaluation with other state-of-the-art algorithms is proposed on synthetic images with several types of generated fog. This evaluation demonstrates that the new algorithm produces similar quality results with homogeneous fog and that it is able to better deal with the presence of heterogeneous fog.
\end{abstract}

\section{INTRODUCTION}

A cause of vehicle accidents is reduced visibility due to bad weather conditions such as fog. This suggests that an algorithm able to improve visibility and contrast in foggy images will be useful for various camera-based Advanced Driver Assistance Systems (ADAS). One may think of an alarm when the distance to the previous vehicle which is observed within the image is too short with respect to the driver's speed. Another possibility is to combine visibility enhancement with pedestrian and two-wheeled vehicles recognition algorithms to deliver adequate alarms. For this kind of ADAS based on the use of a single camera in the vehicle, the contrast enhancement algorithm must be able to process each image in a sequence robustly in real time. The key problem is that, from a single foggy image, contrast enhancement is an ill-posed problem. Indeed, due to the physics of fog, visibility restoration requires to estimate both the scene luminance without fog and the scene depth-map. This implies estimating two unknown parameters per pixel from a single image. Such a problem requires regularization.

The first approach proposed to tackle the visibility restoration problem from a single image is described in [2]. The main idea is to provide interactively an approximate depth-map of the scene geometry allowing to deduce an approximate luminance map without fog. The drawback of this approach for camera-based ADAS is clear: it is not easy to provide the approximate depth-map of the scene geometry from the point of view of the driver all along its road path. In [3], this idea of approximate depth-map was refined by proposing several simple parametric geometric models dedicated to road scenes seen in front of a vehicle. For each type of model, the parameters are fit on each view by maximizing the scene depths globally without producing black pixels in the enhanced image. The limit of this approach is the lack of flexibility of the proposed geometric models.

During the same period of time, another approach to tackle this problem was proposed in [4] based on the use of color images with pixels having a hue different from gray. A difficulty with this kind of approach, for applications we focus on, is that a large part of the image corresponds to the road which is gray and white. Moreover, in our opinion, Intelligent Vehicle applications require visibility enhancement algorithms to be able to process gray-level images.

More recently and for the first time in [5], [6], [1], three visibility enhancement algorithms were proposed working from a single gray-level or color image without using any other extra source of information. These three algorithms rely on a local spatial regularization to solve the problem. The main drawback of [5] and [6] algorithms is their processing time: 5 to 7 minutes and 10 to 20 seconds on a $600 \times 400$ image, respectively. The algorithm proposed in [1] is much faster with a processing time of 0.2 second on a Dual-Core PC on similar image size. The disadvantage of these three visibility enhancement methods is that they are not dedicated to road images and thus the road part of the image which is gray is over-enhanced due to the ambiguity between light colored objects and the presence of fog, as it is illustrated on two images in Fig. 1.

The important property of a road image is that a large part of the image corresponds to the road which can be reasonably assumed to be planar. Visibility enhancement dedicated to planar surface was first proposed in [7], but this algorithm is not able to correctly enhance visibility for the objects out of the road plane. Recently, a visibility enhancement algorithm [8] dedicated to road images was proposed which 

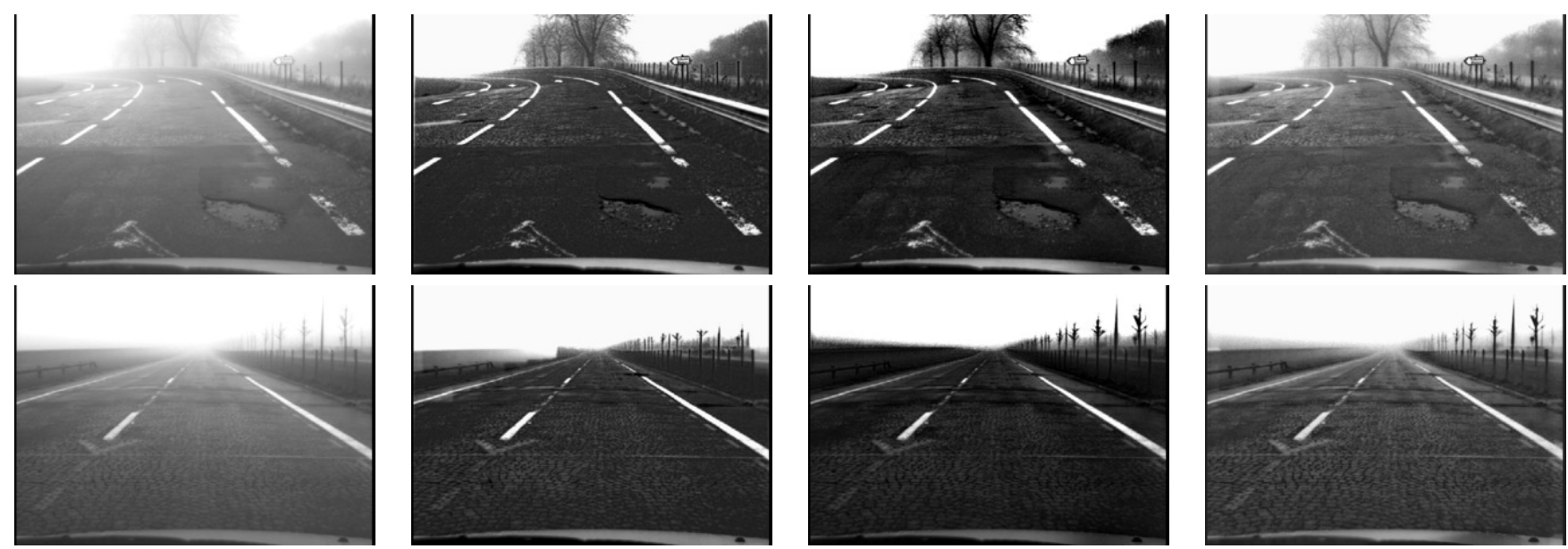

Fig. 1. From left to right, the original image with fog, the results obtain with visibility enhancement using [5], [6] and [1] algorithms. Notice how the road close to the vehicle is over-contrasted or too dark.

was also able to enhance contrast for objects out of the road plane. This algorithm makes good use of the planar road assumption but relies on an homogeneous fog assumption.

In this work, we extend the algorithm described in [1] to take into account that a large part of the image is the planar road. Like [8], the new algorithm makes good use of the planar road assumption and thus can also be seen as the combination of the local visibility enhancement algorithm [1] with the road-specific enhancement algorithm [7]. The proposed algorithm is obtained thanks to our interpretation of [1] as a visibility enhancement algorithm which enforces two constraints, and in particular the no-black-pixel constraint which is not used in [5] and [6]. The main idea in the new algorithm is to take into account the road plane in front of the vehicle, as another third constraint. The obtained algorithm is suitable for camera-based ADAS uses since it is able to process gray-level as well as color images and runs close to real time.

To compare the proposed algorithm to previously presented algorithms, we build up a set of 90 synthetic images with and without fog. The algorithms are applied on foggy images and results are compared with the images without fog.

The article is structured as follows. Section II presents the fog model we use. In section III, the multiscale retinex algorithm (MSR) [9] is summarized. In section IV, different approaches of visibility enhancement are described: based on the planar assumption (PA) [7], based on a free-space segmentation (FSS) [8], based on no-black-pixel constraint (NBPC) [1], and finally the new combined algorithm named NBPC+PA. In section V is provided a comparison between MSR, FSS, NBPC and NBPC+PA algorithms based on a quantitative evaluation on $18 \times 4$ color images, illustrating the properties of each algorithm.

\section{EFFECT OF FOG}

Assuming an object of intrinsic luminance $L_{0}(u, v)$, its apparent luminance $L(u, v)$ in presence of a fog of extinction coefficient $k$ is modeled by Koschmieder's law [10]:

$$
L(u, v)=L_{0}(u, v) e^{-k d(u, v)}+L_{s}\left(1-e^{-k d(u, v)}\right)
$$

where $d(u, v)$ is the distance of the object at pixel $(u, v)$ and $L_{S}$ is the luminance of the sky. As described by (1), fog has two effects: first an exponential decay $e^{-k d(u, v)}$ of the intrinsic luminance $L_{0}(u, v)$, and second the addition of the luminance of the atmospheric veil $L_{S}\left(1-e^{-k d(u, v)}\right)$ which is an increasing function of the object distance $d(u, v)$.

From now on, we assume that camera response is linear, and thus image intensity $I$ is substituted to luminance $L$.

\section{Multiscale Retinex (MSR)}

We now recall the retinex algorithm. The retinex is not a Visibility enhancement algorithm since it is not based on Koschmieder's law (1), but it is able to remove a constant intensity on the image. However, we found interesting to include it in our comparison to quantify the gain visibility enhancement algorithms are able to achieve.

The multiscale retinex (MSR) is a non-linear image enhancement algorithm proposed by [9]. The overall impact is to brighten up areas of poor contrast/brightness but not at the expense of saturating areas of good contrast/brightness. The MSR output is simply the weighted sum of the outputs of several single scale retinex (SSR) at different scales. Each SSR is capable of enhancing some particular characteristic of the input image. For instance, narrow surrounds highlight the fine features but almost all tonal rendition is lost. Wide surrounds retain all the tonal information but do not enhance the small fine features. Hence, multiple surrounds are needed to achieve a graceful balance between dynamic range compression and tonal rendition.

Each color component being processed independently, the basic form of the SSR for on input image $I(u, v)$ is:

$$
R_{k}(u, v)=\log I(u, v)-\log \left[F_{k}(u, v) * I(u, v)\right]
$$

where $R_{k}(u, v)$ is the SSR output, $F_{k}$ represents the $k^{t h}$ surround function, and $*$ is the convolution operator. The 
surround functions, $F_{k}$ are given as normalized Gaussians:

$$
F_{k}(u, v)=\kappa_{k} e^{-\left(u^{2}+v^{2}\right) / \sigma_{k}^{2}}
$$

where $\sigma_{k}$ is the scale controlling the extent of the surround and $\kappa_{k}$ is for unit normalization. Finally the MSR output is:

$$
\left.R(u, v)=\sum_{k=1}^{k=K} W_{k} R_{k}(u, v)\right)
$$

where $W_{k}$ is the weight associated to $F_{k}$.

The number of scales used for the MSR is, of course, application dependent. We have tested different sets of parameters, and we did not found a better parametrization than the one proposed by [11]. It consists of three scales representing narrow, medium, and wide surrounds that are sufficient to provide both dynamic range compression and tonal rendition: $K=3, \sigma_{1}=15, \sigma_{2}=80, \sigma_{3}=250$, and $W_{k}=1 / 3$ for $k=1,2,3$.

Results obtained using the multiple retinex on three foggy images are presented in column two of Fig. 2.

\section{VISIBILITY ENHANCEMENT}

Three visibility enhancement algorithms are now presented: enhancement assuming a planar scene assumption (PA), enhancement with free-space segmentation (FSS) and enhancement with no-black-pixel constraint (NBPC). The advantages and limits of these three algorithms are discussed and a new algorithm is proposed we named NBPC+PA algorithm which tries to combine advantages of PA and NBPC algorithms. The results obtained by the four algorithms are presented in Fig. 2 on three images.

\section{A. With the planar assumption (PA)}

Dedicated to in-vehicle applications, the algorithm proposed in [12], [10] is able to detect the presence of fog and to estimate the visibility distance which is directly related to $k$ in Koschmieder's law (1). This algorithm, also known as the inflexion point algorithm, mainly relies on three assumptions: homogeneous fog, the main part of the image displays the road surface which is assumed to be a planar and homogeneous surface. From the estimated fog parameters, the contrast in the road part of the image can be restored as explained in [7].

Using the planar road surface assumption and knowing the approximate camera calibration with respect to the road, it is possible to associate a distance $d$ with each line $v$ of the image:

$$
d=\frac{\lambda}{v-v_{h}} \quad \text { if } v>v_{h}
$$

where $v_{h}$ is the vertical position of the horizon line in the image and $\lambda$ depends on intrinsic and extrinsic parameters of the camera, see [10] for details.

Using the assumption of a road with homogeneous photometric properties $\left(I_{0}\right.$ is constant), fog can be detected and the extinction coefficient of the atmosphere $k$ can be estimated using Koschmieder's law (1). After substitution of $d$ given by (5), (1) becomes:

$$
I(v)=I_{0} e^{-k \frac{\lambda}{v-v_{h}}}+I_{S}\left(1-e^{-k \frac{\lambda}{v-v_{h}}}\right)
$$

By twice taking the derivative of $I$ with respect to $v$, the following is obtained:

$$
\frac{d^{2} I}{d v^{2}}(v)=k \frac{\lambda\left(I_{0}-I_{s}\right)}{\left(v-v_{h}\right)^{3}} e^{-k \frac{\lambda}{v-v_{h}}}\left(\frac{k \lambda}{v-v_{h}}-2\right)
$$

The equation $\frac{d^{2} I}{d v^{2}}=0$ has two solutions. The solution $k=0$ is of no interest. The only useful solution is given by (8):

$$
k=\frac{2\left(v_{i}-v_{h}\right)}{\lambda}
$$

where $v_{i}$ denotes the position of the inflection point of $I(v)$. The value of $I_{s}$ is obtained as the intensity of the sky, i.e most of the time it corresponds to the maximum intensity in the image. Having estimated the value of $k$ and $I_{s}$, the pixels on the road plane can be restored as $R(u, v)$ by reversing Koschmieder's law [7]:

$$
R(u, v)=I(u, v) e^{k \frac{\lambda}{v-v_{h}}}+I_{S}\left(1-e^{k \frac{\lambda}{v-v_{h}}}\right)
$$

As in [3], the introduction of a clipping plane in the equation of the distance (5) allows, still using the reverse of Koschmieder's law, to enhance visibility and contrast in the whole image. More precisely, the used geometrical model consists in the road plan (5) in the bottom part of the image, and in a plan in front of the camera in the top part of the image. The height of the line which separates the road model and the clipping plane is denoted $c$. As a consequence only large distances are clipped. In summary, the geometrical model $d_{c}(u, v)$ of a pixel at position $(u, v)$ is expressed as:

$$
d_{c}(u, v)=\left\{\begin{array}{l}
\frac{\lambda}{\left(v-v_{h}\right)} \text { if } v>c \\
\frac{\lambda}{\left(c-v_{h}\right)} \text { if } v \leq c
\end{array}\right.
$$

Results obtained with the previous model where the clipping plane is set at the visibility distance are shown on three foggy images in column three of Fig. 2. From these results it appears that the road part of the image is correctly restored.

\section{B. With free-space segmentation (FSS)}

To be able to enhance the visibility in the rest of the scene, an estimate of the depth $d(u, v)$ of each pixel is needed. In [3], a parameterized 3D model of a road scene was proposed with a reduced number of geometric parameters. Even if the proposed models are relevant for most road scenes and even if the parameters of the selected model are optimized to achieve best enhancement without black pixel in the resulting image, the proposed models are not generic enough to handle all traffic configurations.

In [8], a different scheme is proposed which consists in assuming once again that the road is planar with a clipping plane, see (10). When geometric model (10) is assumed, the contrast of objects belonging to the road plane is correctly 

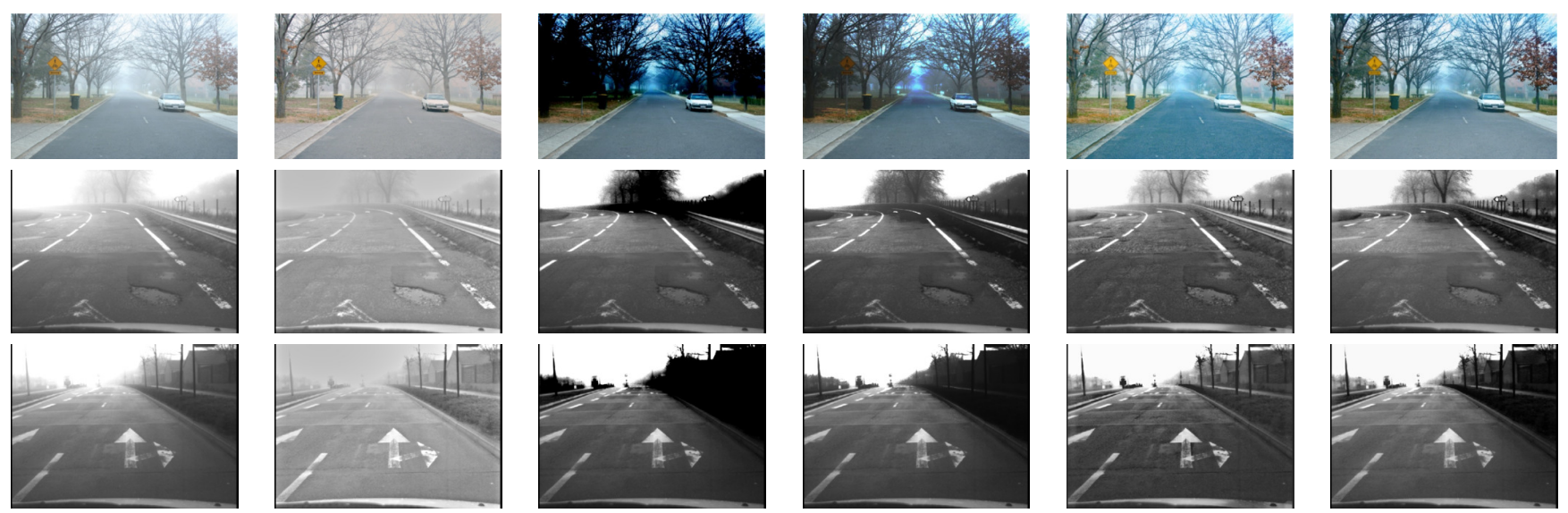

Fig. 2. From left to right, the original image with fog, the images enhanced using algorithms: retinex, planar assumption with clipping, free-space segmentation, no-black-pixel constraint and no-black-pixel constraint combined with planar assumption.

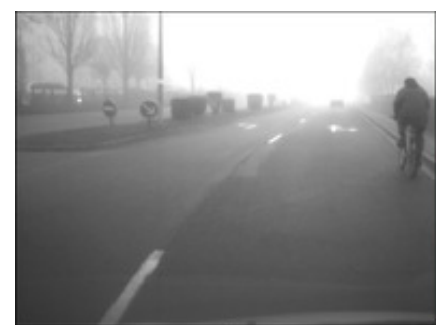

(a)

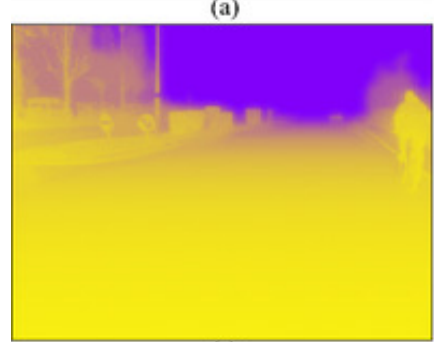

(c)

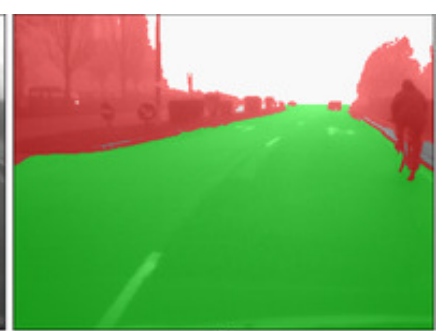

(b)

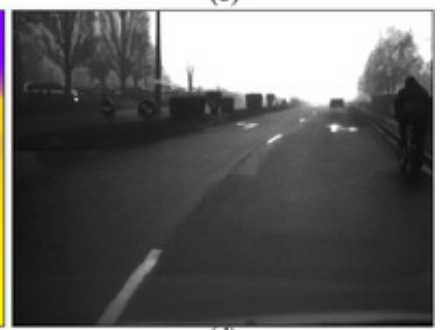

(d)

$c$ is close to the line of horizon, the clipping plane is far from the camera and the visibility is only slightly enhanced. The larger the value of $c$, the closer the clipping plane is to the camera, and thus the stronger the enhancement. The enhancement in (9) can be so strong that enhanced intensity becomes negative.

Every $c_{\min }(u, v)$ value can be associated to a distance $d_{\min }(u, v)$ using (10). The obtained depthmap on the foggy image of Fig. 3(a) is displayed in Fig. 3(c). Then, a rough estimate of the depthmap $d(u, v)$ is obtained as a fixed percentage $p$ of depth map $d_{\text {min }}(u, v)$. Percentage $p$ specifies the strength of the enhancement and is usually set to $90 \%$ for this method. The obtained depthmap is used to enhance the contrast on the whole image using reversed Koschmieder's law as illustrated in Fig. 3(d). The algorithm is detailed in [8], [13] and other results are shown in the fourth column of Fig. 2.

Fig. 3. Steps of visibility enhancement with FSS algorithm: (a) original image, (b) segmentation of vertical objects (in red) and free-space region (in green), (c) rough estimate of the scene depthmap, (d) obtained visibility enhancement.

restored, as seen in previous section. Conversely, the contrast of vertical objects of the scene (vehicles, trees,...) is falsely restored since their depth in the scene is largely overestimated. Consequently, their restored intensity using (9) are negative and thus are set to zero in the enhanced image. These are named black pixels. The set of the black pixels gives a segmentation of the image in the objects out and in the road plane and this allows to deduce the free-space region $D$, as illustrated in green and red in Fig. 3(b), see [13] for details.

For each pixel in the free-space region $D$, the road plane model (5) is correct. For pixels out of the road plane (red region in Fig. 3(b)), it is proposed in [8] to use the geometric model (10) and, for each pixel, to search for the smallest $c$ which leads to a positive intensity in the restored image. The obtained values are denoted $c_{\min }(u, v)$. Indeed, when

\section{With no-black-pixel constraint (NBPC)}

In [1], an algorithm which relies on a local regularization is also proposed. As explained in [1], the distance $d(u, v)$ being unknown, the objective of the visibility enhancement in a single image can be set as inferring the intensity of the atmospheric veil $V(u, v)=I_{s}\left(1-e^{-k d(u, v)}\right)$. Most of the time, the intensity of the sky $I_{s}$ corresponds to the maximum intensity in the image, and thus $I_{s}$ can be set to 1 without loss of generality, assuming the input image normalized. After substitution of $V$ in (1) and with $I_{s}=1$, Koschmieder's law is rewritten as:

$$
I(u, v)=I_{0}(u, v)(1-V(u, v))+V(u, v)
$$

The foggy image $I(u, v)$ is enhanced again as $R(u, v)$ simply by the reversing of (11):

$$
R(u, v)=\frac{I(u, v)-V(u, v)}{1-V(u, v)}
$$

One may notice that the enhancement equation provided by Koschmieder's law is a linear transformation. Interestingly, it gives us the exact link between its intercept and its slope. 
The atmospheric veil $V(u, v)$ being unknown, let us enumerate the constraints $V(u, v)$ is subject to. $V(u, v)$ must be higher or equal to zero and $V(u, v)$ is lower than $I(u, v)$ :

$$
0 \leq V(u, v) \leq I(u, v)
$$

These are the photometric constraints as named in [5].

We now introduce a new constraint which focuses on the reduction of the number of black pixels in the enhanced image $R$. This constraint is named no-black-pixel constraint and states that the local standard deviation of the enhanced pixels around a given pixel position must be lower than its local average:

$$
\operatorname{std}(R) \leq \bar{R}
$$

In case of a Gaussian distribution of the intensities, this criterion implies $15.8 \%$ of the intensities becoming black. Using $2 \operatorname{std}(R)$ instead of $\operatorname{std}(R)$ leads to a stronger criterion where only $2.2 \%$ of the intensities become black.

The difficulty with this last constraint is that it is set as a function of the unknown result $R$. Thanks to the linearity of (12), the no-black-pixel constraint can be turned into a constraint involving $V$ and $I$ only. For this purpose, we now enforce local spatial regularization by assuming that locally around pixel position $(u, v)$, the scene depth is constant and the fog homogeneous, i.e equivalently, the atmospheric veil locally equals $V(u, v)$ at the central position. Under this assumption, we derive using (12) that the local averages $\bar{I}$ and $\bar{R}$ are related by $\bar{R}=\frac{\bar{I}-V(u, v)}{1-V(u, v)}$ and that the standard deviations $s t d(I)$ and $s t d(R)$ are related by $s t d(R)=\frac{s t d(I)}{1-V(u, v)}$. We therefore obtain, after substitution of the two previous results in (14), the no-black-pixel constraint rewritten as a function of $V(u, v)$ and $I$ :

$$
V(u, v) \leq \bar{I}-\operatorname{std}(I)
$$

The atmospheric veil $V(u, v)$ is set as a percentage $p$ of the minimum over the two previous upper bounds:

$$
V(u, v)=p \min (I(u, v), \bar{I}-s t d(I))
$$

Percentage $p$ specifies the strength of the enhancement and is usually set to $95 \%$ for this method. The enhanced image is obtained by applying (12) using the previous $V$. The algorithm derived from the photometric and no-black-pixel constraints turns out to be the one described in [1] where $\bar{I}$ is obtained as the median of the local intensities and the standard deviation as the median of the absolute differences between the intensities and $\bar{I}$.

This enhancement algorithm is presented with a graylevel input image but can be extended easily to color images $(r(u, v), g(u, v), b(u, v))$ by using $I(u, v)=$ $\min (r(u, v), g(u, v), b(u, v))$ for the input gray-level image, in the previous equations after adequate white balance. The obtained $V$ gives the amount of white that must be subtracted to the three color channels. The complete algorithm is available in Matlab $^{T M}$ at perso.lcpc.fr/tarel.jean-philippe/visibility/.

Fig. 2 shows the visibility enhancement obtained by the NBPC algorithm in the fifth column. One can notice that the contrast on the road part of the resulting image is too much enhanced. This is due to the fact that the atmospheric veil $V(u, v)$ in the road part of the image is over-estimated. This is a consequence of the locality property of the NBPC algorithm.

\section{Combining the no-black-pixel constraint and the planar assumption $(\mathrm{NBPC}+\mathrm{PA})$}

As explained in the previous section, the visibility enhancement with NBPC is a generic local method which is not dedicated to road images and which is in difficulty in presence of a large uniform region such as the road. In the visibility enhancement with FSS, as explained in section IV$\mathrm{B}$, a segmentation is performed to split the image into three regions: the sky, the objects out of the road plane, and the free-space in the road plane, and different enhancement processes are performed depending on the region. The difficulty with an approach based on segmentation is to manage correctly the transition between the regions. An alternative to the segmentation, when the problem is set as the inference of the atmospheric veil $V(u, v)$, is to introduce a third constraint which prevents over-estimation in the bottom part of the image. Indeed, the road being gray, the upper bound given by the NBPC in the bottom part of the image is usually large when the atmospheric veil cannot be large, due to the reduced distance between the camera and the road.

In practice, it is very rare to observe fog with a meteorological visibility distance lower than $50 \mathrm{~m}$. The meteorological visibility distance $d_{m}$ is related to extinction coefficient $k$ by $d_{m}=-\frac{\ln (0.05)}{k}$, see [10]. As a consequence, from $d_{m} \geq 50$, we deduce $k \leq-\frac{\ln (0.05)}{50}$. With the assumption that the road is a plane until a certain distance, and that the camera calibration is known with respect to the road, $\lambda$ and $v_{h}$ are known, and thus, using the last term of equation (6), we define the third constraint the atmospheric veil is subject to:

$$
V(u, v) \leq I_{S}\left(1-e^{\frac{\ln (0.05) \lambda}{d_{\min }\left(v-v_{h}\right)}}\right)
$$

where $d_{\min }=50 \mathrm{~m}$ as justified previously. We named (16) the planar assumption constraint. Like in the NBPC algorithm, the atmospheric veil $V(u, v)$ is set as a percentage $p$ of the minimum over the now three upper bounds:

$$
V(u, v)=p \min \left(I(u, v), \bar{I}-s t d(I), I_{s}\left(1-e^{\frac{\ln (0.05) \lambda}{d_{\min }\left(v-v_{h}\right)}}\right)\right)
$$

The enhanced image results of the application of (12) using the previous $V$. We named it visibility enhancement with NBPC+PA.

In the presence of fog with a meteorological visibility distance lower than $d_{\text {min }}=50 \mathrm{~m}$, this third constraint limits the possibilities of enhancement which will be partial at short distances even with $p=100 \%$. An alternate approach, not tested here, is to run the fog detection algorithm and the $k$ estimation as explained in section IV-A and to use the estimated $k$ in (16) instead of $-\frac{\ln (0.05)}{50}$. This refinement should lead to more accurate results compared the NBPC+PA when the fog is uniform but it also may lead to a bias when the fog is not homogeneous enough. 

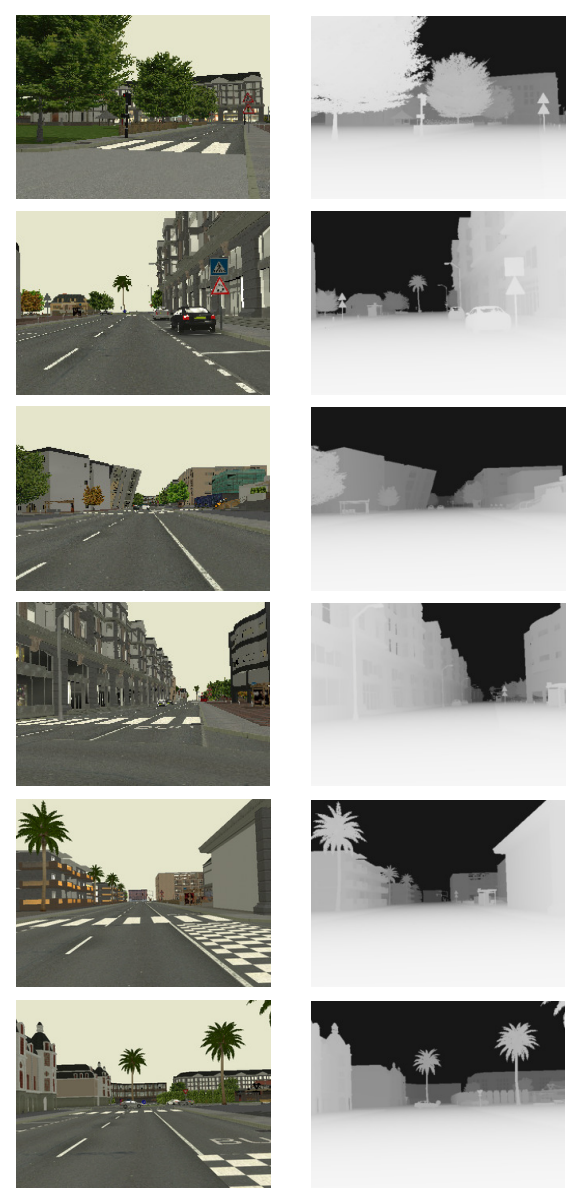
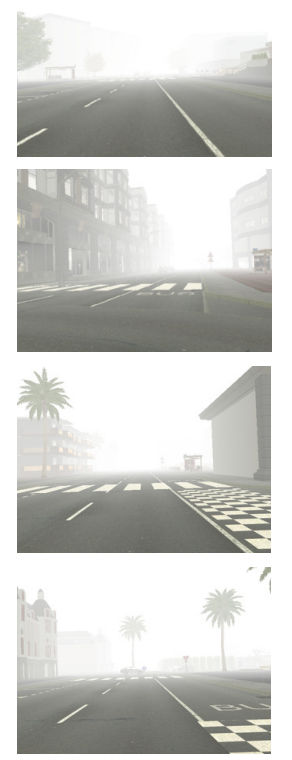
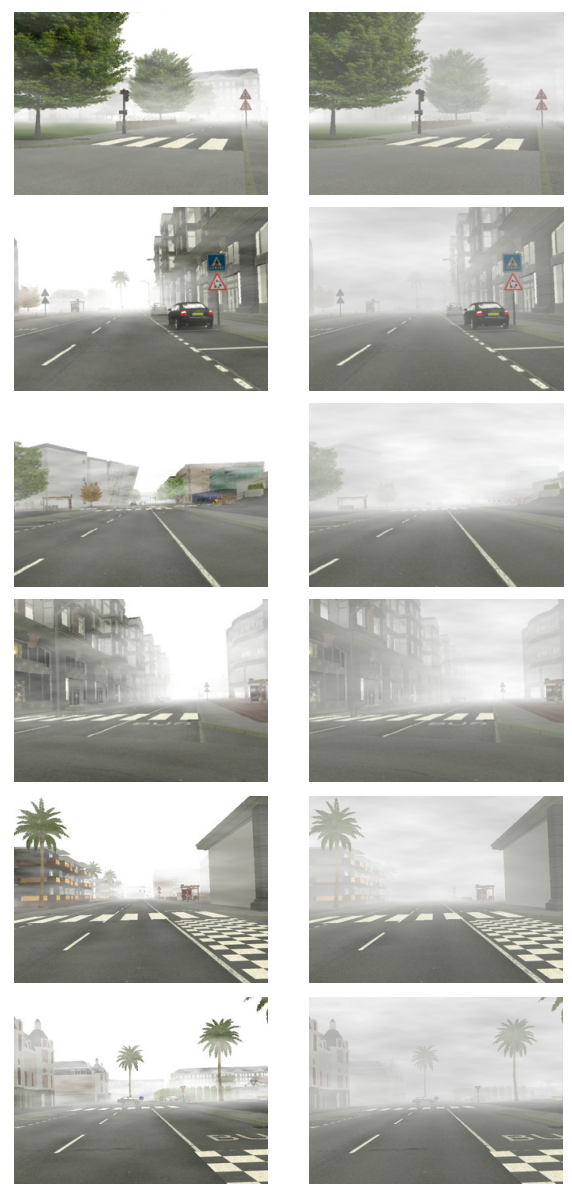
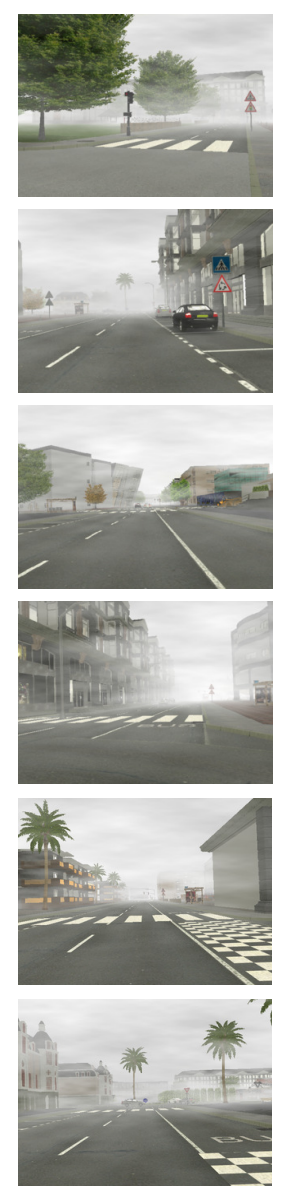

Fig. 4. First column is the original synthetic image. Second column is the depth map. Third to sixth columns are the original image with different types of synthetic fog added, from left to right: uniform fog, heterogeneous $k$ fog, heterogeneous $L_{s}$ fog, and heterogeneous $k$ and $L_{s}$ fog.

\section{EXPERIMENTS}

To evaluate visibility enhancement algorithms, we need images of the same scene with and without fog. However, obtaining such kind of pairs of images is extremely difficult in practice since it requires to check that the illumination conditions are the same into the scene with and without fog. As a consequence, for the evaluation of the proposed visibility enhancement algorithm and its comparison with existing algorithms, we build up a set of synthetic images with and without fog.

\section{A. Synthetic images}

The software we used is named $\mathrm{SiVIC}^{T M}$ and allows to build physically-based road environments, to generate a moving vehicle with a physically-driven model of its dynamic behavior [14], and virtual embedded sensors (proprioceptive, exteroceptive and communication). From a realistic complex urban model, we produced images from a virtual camera inboard a simulated vehicle moving on a road path. We have generated a set of 18 images from various view points trying to sample as many scene aspects as possible. Each image is of size $640 \times 480$ and a subset of 6 images is shown in the first column of Fig. 4. For each point of view, its associated depthmap is also generated, as shown in the second column of Fig. 4. Indeed, the depthmap is required to be able to add fog consistently in the images. We generate 4 different types of fog:

- Uniform fog: Koschmieder's law (1) is applied on the 18 original images with a visibility distance of $85.6 \mathrm{~m}$ to generate 18 images with a uniform fog added.

- Heterogeneous $k$ fog: fog being not always perfectly homogeneous, we introduce variability in Koschmieder's law (1) by weighting differently $k$ with respect to the pixel position. These spatial weights are obtained as a Perlin's noise between 0 and 1 , i.e a noise spatially correlated at different scales $(2,4,8, \cdots$ to the size of the image in pixels) [15]. Perlin's noise is obtained as a linear combination over the spatially correlated noise generated at different scales with weight $\log _{2}(s)^{2}$ for scale $s$.

- Heterogeneous $L_{s}$ fog: rather than having $k$ heterogeneous and $L_{s}$ constant, we also test the case where $L_{s}$ is heterogeneous thanks again to Perlin's noise and where $k$ is constant.

- Heterogeneous $k$ and $L_{s}$ fog: in order to challenge the algorithms, we also generate a fog based on Koschmieder's law (1) where $k$ and $L_{s}$ are both heterogeneous thanks to two independent Perlin's noises. 


\begin{tabular}{|c|c|c|c|c|}
\hline Algorithm & Uniform & Variable $k$ & Variable $L_{s}$ & Variable $k \& L_{s}$ \\
\hline Nothing & $70.6 \pm 5.3$ & $49.9 \pm 4.9$ & $56.9 \pm 5.3$ & $39.1 \pm 4.9$ \\
MSR & $46.0 \pm 4.5$ & $71.4 \pm 14.3$ & $46.4 \pm 5.0$ & $71.1 \pm 13.6$ \\
FSS & $34.7 \pm 6.3$ & $34.1 \pm 3.0$ & $44.2 \pm 6.6$ & $43.8 \pm 9.1$ \\
NBPC & $48.8 \pm 5.8$ & $35.5 \pm 6.4$ & $42.9 \pm 5.9$ & $35.5 \pm 6.2$ \\
NBPC+PA & $31.9 \pm 4.6$ & $29.0 \pm 4.3$ & $40.2 \pm 4.3$ & $37.2 \pm 4.4$ \\
\hline
\end{tabular}

TABLE I

AVERAGE ABSOLUTE DIFFERENCE ON 18 IMAGES BETWEEN ENHANCED IMAGES AND TARGET IMAGES WITHOUT FOG, FOR THE 4 COMPARED ALGORITHMS, AND FOR THE 4 TYPES OF SYNTHETIC FOG.

Finally the test image database contains 4 sets of 18 foggy images associated with the 18 original images. Examples of foggy images computed as previously described are displayed in the last four columns of Fig. 4. Notice the differences in complexity between the different types of generated fog.

\section{B. Comparison}

We apply each algorithm on the 4 types of synthetic fog. Used algorithms are: multiscale retinex (MSR), enhancement with free-space segmentation (FSS), enhancement with noblack-pixel constraint (NBPC) and enhancement with noblack-pixel constraint combined with planar scene assumption (NBPC+PA). The results on 6 images with a uniform fog is presented in Figure 5. Notice the increase of the contrast for the farther objects: some object that was barely visible in foggy image appears clearly in enhanced images. A first visual analysis confirms that MSR is not suited for foggy images, that vertical objects appears too dark with FSS, that roads look over-corrected by NBPC, and that NBPC+PA comes as a nice trade-off.

The quantified comparison consists simply in computing the absolute difference between the image without fog and the image obtained after enhancement. Results, averaged over the 18 images, the number of image pixels and the number of image color components, are shown in Tab. I.

To qualify easily the improvement obtained by the different algorithms, the average absolute difference between the images with and without fog is also computed and shown in column two of the table. One can notice that the proposed algorithms are able, in the best case, to divide the average difference by approximatively a factor of two.

The multiscale retinex (MSR) is not a visibility enhancement algorithm dedicated to scene with various object depths. The average difference is decreased for the uniform fog and for fog with heterogeneous $L_{s}$. Interestingly, when the $k$ is heterogeneous, the multiscale retinex is worse than doing nothing. This is explained by the fact that MSR increases some contrasts corresponding to fog and not to the scene.

With uniform fog, enhancement with free-space segmentation (FSS) and with no-black-pixel constraint combined with planar scene assumption (NBPC+PA) gives best results, while enhancement with no-black-pixel constraint (NBPC) is worse than the multiscale retinex (MSR), due to too strong contrast distortions on the road part of the image.
Nevertheless enhancement with NBPC is better than MSR at long range distances. The enhancement with NBPC+PA allows to keep the good properties of the enhancement with NBPC at long range distances without contrast distortions on the road part of the image thanks to the combination with the planar assumption.

For the three types of heterogeneous fog, enhancement with NBPC+PA leads to better results compared to FSS. This can be explained by the fact that FSS enhancement algorithm relies strongly on the assumption that $k$ and $L_{s}$ are constant over whole image when NBPC+PA algorithm does not. Indeed, NBPC+PA algorithm only assumes that $k$ and $L_{s}$ are locally constant in the image and thus, most of the time, it performs better with heterogeneous fog compared to others.

\section{CONCLUSION}

Thanks to the interpretation of the local visibility enhancement algorithm [1] in terms of two constraints on the inference of the atmospheric veil, we introduce a third constraint to take into account the fact that road images contain a large part of planar road, assuming a fog with a visibility distance higher than $50 \mathrm{~m}$. The obtained visibility enhancement algorithm performs better than the original algorithm on road images as demonstrated on a set of 18 synthetic images where a uniform fog is added following Koschmieder's law. We also generate different types of heterogeneous fog, a situation never considered previously in our domain. The proposed algorithm also demonstrates its ability to improve visibility in such difficult heterogeneous situations. Obtained results are compared with respect to state-of-the-art algorithms: multiscale retinex [9], enhancement with free-space segmentation (FSS) [8] and enhancement based on no-blackpixel constraint (NBPC) [1].

We are planning to extend the set of synthetic images used for the ground truth and to make it available on www.lcpc.fr/en/produits/fog/ for research purpose and in particular to allow other researchers to rate their own visibility enhancement algorithms.

\section{ACKNOWLEDGMENTS}

Thanks to Robby T. Tan for providing the two images of the second column of Fig. 1 and the first image of Fig. 2, to Kaiming He for providing the two images of the third column of Fig. 1, and to Fabrice Neyret for its suggestions about fog synthesis. This work is partly funded by the ANR (French National Research Agency) within the ICADAC project (6866C0210).

\section{REFERENCES}

[1] J.-P. Tarel and N. Hautière, "Fast visibility restoration from a single color or gray level image," in Proceedings of IEEE International Conference on Computer Vision (ICCV'09), Kyoto, Japan, 2009, pp. 2201-2208, http://perso.lcpc.fr/tarel.jean-philippe/publis/iccv09.html.

[2] S. G. Narashiman and S. K. Nayar, "Interactive deweathering of an image using physical model," in IEEE Workshop on Color and Photometric Methods in Computer Vision, Nice, France, 2003. 

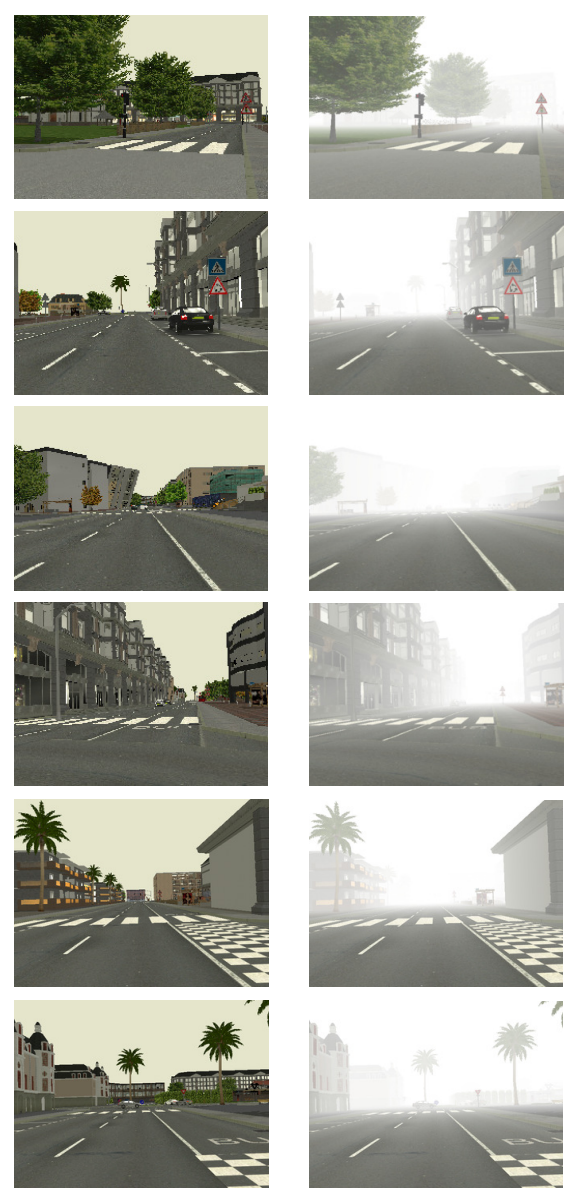
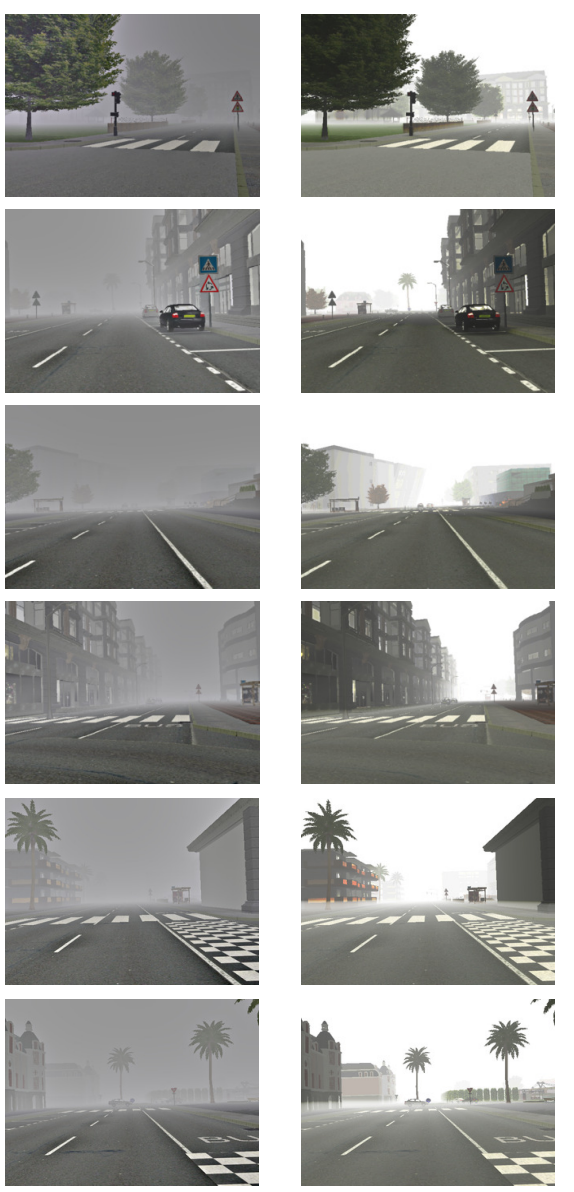
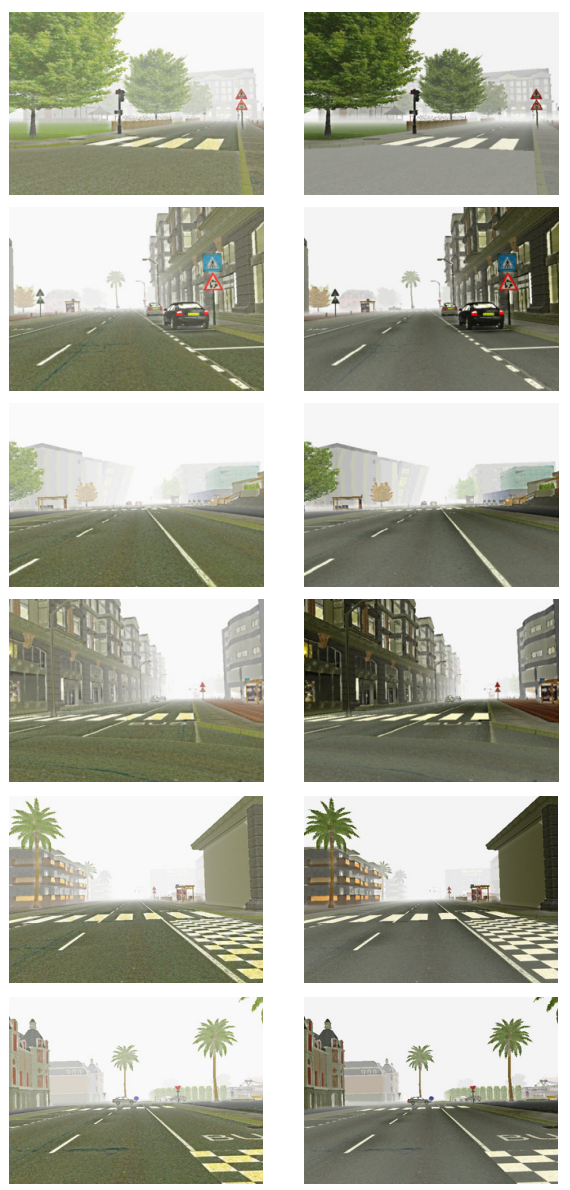

Fig. 5. From left to right, the original image without fog, the processed image with uniform fog, the images enhanced using multiscale retinex, free-space segmentation, no-black-pixel constraint and no-black-pixel constraint combined with planar scene assumption.

[3] N. Hautière, J.-P. Tarel, and D. Aubert, "Towards fog-free invehicle vision systems through contrast restoration," in IEEE International Conference on Computer Vision and Pattern Recognition (CVPR'07), Minneapolis, Minnesota, USA, 2007, pp. 1-8, http://perso.lcpc.fr/tarel.jean-philippe/publis/cvpr07.html.

[4] R. Tan, N. Pettersson, and L. Petersson, "Visibility in bad weather from a single image," in Proceedings of the IEEE Intelligent Vehicles Symposium (IV'07), Istanbul, Turkey, 2007, pp. 19-24.

[5] R. Tan, "Visibility in bad weather from a single image," in IEEE Conference on Computer Vision and Pattern Recognition (CVPR'08), Anchorage, Alaska, 2008, pp. 1-8.

[6] K. He, J. Sun, and X. Tang, "Single image haze removal using dark channel prior," in IEEE Conference on Computer Vision and Pattern Recognition (CVPR'09), Miami Beach, Florida, USA, 2009, pp. 19561963.

[7] N. Hautière and D. Aubert, "Contrast restoration of foggy images through use of an onboard camera," in Proc. IEEE Conference on Intelligent Transportation Systems (ITSC'05), Vienna, Austria, 2005, pp. 1090-1095.

[8] N. Hautière, J.-P. Tarel, and D. Aubert, "Mitigation of visibility loss for advanced camera based driver assistances," To appear in IEEE Transactions on Intelligent Transportation Systems, 2010.

[9] Z. Rahman, D. J. Jobson, and G. W. Woodell, "Multiscale retinex for color rendition and dynamic range compression," in Society of PhotoOptical Instrumentation Engineers (SPIE) Conference Series, ser. Presented at the Society of Photo-Optical Instrumentation Engineers (SPIE) Conference, A. G. Tescher, Ed., vol. 2847, Nov. 1996, pp. 183-191.
[10] N. Hautière, J.-P. Tarel, J. Lavenant, and D. Aubert, "Automatic fog detection and estimation of visibility distance through use of an onboard camera," Machine Vision and Applications, vol. 17, no. 1, pp. 8-20, 2006, http://perso.lcpc.fr/tarel.jean-philippe/publis/mva06.html.

[11] Z. Rahman, D. J. Jobson, G. A. Woodell, and G. D. Hines, "Image enhancement, image quality, and noise," in Society of Photo-Optical Instrumentation Engineers (SPIE) Conference Series, ser. Presented at the Society of Photo-Optical Instrumentation Engineers (SPIE) Conference, K. M. Iftekharuddin \& A. A. S. Awwal, Ed., vol. 5907, Aug. 2005, pp. 164-178.

[12] J. Lavenant, J.-P. Tarel, and D. Aubert, "Procédé de détermination de la distance de visibilité et procédé de détermination de la présence d'un brouillard," French pattent number 0201822, INRETS/LCPC, February 2002.

[13] N. Hautière, J.-P. Tarel, and D. Aubert, "Free space detection for autonomous navigation in daytime foggy weather," in Proceedings of IAPR Conference on Machine Vision Applications (MVA'09), Yokohama, Japan, 2009, pp. 501-504, http://perso.lcpc.fr/tarel.jeanphilippe/publis/mva09.html.

[14] D. Gruyer, C. Royere, N. du Lac, G. Michel, and J.-M. Blosseville, "Sivic and rtmaps, interconnected platforms for the conception and the evaluation of driving assistance systems," in Proc. Intelligent Transport Systems World Congress, London, England, 2006, pp. 1-8.

[15] K. Perlin, "An image synthesizer," SIGGRAPH Computer Graphic, vol. 19 , no. 3, pp. 287-296, 1985. 